\section{Aportes para el monitoreo participativo de la calidad del agua del río Tacuarembó en el noreste uruguayo}

Patricia Iribarne

iribarne@fcien.edu.uy

(iD) orcid.org/0000-0003-4833-1477

\author{
María Laura Lavaggi \\ maria.lavaggi@cur.edu.uy \\ (iD) orcid.org/0000-0002-4224-1799
}

Universidad de la República, Uruguay.

RECEPCIÓN: 29/04/21

ACEPTACIÓN FINAL: 09/06/21

\section{Resumen}

Implementar procesos participativos para involucrar a diferentes actores sociales vinculados a problemas ambientales locales puede contribuir a mejorar la comprensión de las causas de estos y empoderar a los ciudadanos frente a la toma de decisiones colectivas. La importancia de esta participación ciudadana se refleja en la experiencia llevada a cabo en Tranqueras (Rivera, Uruguay), donde se convocó a distintos actores para efectuar un monitoreo participativo de la calidad de agua del río Tacuarembó. El proceso implicó la realización de talleres para la visualización de problemáticas ambientales y el diseño de un muestreo, colecta participativa y análisis de la calidad del agua. Esta experiencia generó un espacio de intercambio de saberes entre la comunidad y la Universidad que conduce a la reflexión sobre la relevancia de la dimensión epistemológica de los problemas ambientales, la forma en que se llega a su conocimiento y las distintas perspectivas con las que se abordan.

Palabras clave: investigación participativa; extensión universitaria, políticas públicas problemas ambientales; desarrollo local.
Contributions for the participatory monitoring of the water quality of the Tacuarembó river in northeastern Uruguayan

\section{Abstract}

Implementing participatory processes to involve different social actors linked to local environmental problems can be helpful to improve the understanding of their causes and to empower citizens facing collective decisionmaking. The importance of this citizen participation is reflected in the experience carried out in Tranqueras (Rivera, Uruguay) where different actors were summoned to carry out participatory monitoring of the water quality of the Tacuarembó river. The process involved several workshops for the visualization of environmental problems and the design of a participatory sampling and analysis of water quality. This experience generated a teachinglearning space between the community and the University that raises the reflection of the relevance of the epistemological dimension of environmental problems and the way in which their knowledge is generated and the different perspectives of how environmental problems are addressed.

Keywords: participatory research; university extension, public policies; environmental problems; local development.
Ambiente y extensión universitaria /

Intervenciones

두(이잉

\author{
Contribuições para o monitoramento \\ participativo da qualidade da água do rio \\ Tacuarembó no nordeste do Uruguai
}

\section{Resumo}

A implementação de processos participativos para envolver diferentes atores sociais ligados a problemas ambientais locais pode ajudar a melhorar a compreensão de suas causas e capacitar os cidadãos para a toma de decisões coletivas. A importância dessa participação cidadã é mostrada na experiência realizada em Tranqueras (Rivera, Uruguai), onde diferentes atores foram convocados para realizar o monitoramento participativo da qualidade da água do rio Tacuarembó. O processo envolveu a realização de oficinas para a visualização de problemas ambientais e o desenho de uma amostragem, coleta participativa e análise da qualidade da água. Essa experiência gerou um espaço de ensino-aprendizagem entre a comunidade e a Universidade que suscita a reflexão sobre a relevância da dimensão epistemológica dos problemas ambientais, a maneira como seus conhecimentos são gerados e as diferentes perspectivas com as quais os problemas ambientais são abordados.

Palavras-chave: pesquisa participativa; extensão universitária, políticas públicas; problemas ambientais; desenvolvimento local.

Para citación de este artículo: Iribarne, P. y Lavaggi M. L. (2021). Aportes para el monitoreo participativo de la calidad del agua del río Tacuarembó en el noreste uruguayo. +E: Revista de Extensión Universitaria, 11(14), e0009. doi: 10.14409/extension.2021.14.Ene-Jun.e0009 


\section{Introducción}

Esta investigación tiene como objetivo aportar insumos para el monitoreo participativo de cuerpos de agua del noreste uruguayo a la vez de generar un espacio de reflexión sobre la importancia de la participación de actores locales en la definición y abordaje de problemáticas ambientales. Para ello, se describe, analiza y reflexiona sobre la base de una experiencia llevada a cabo en la ciudad de Tranqueras (Rivera, Uruguay) durante el período 2016-2017, donde se convocó a distintos actores para realizar un monitoreo participativo de la calidad de agua del río Tacuarembó en un tramo de $6 \mathrm{~km}$, aproximadamente, que atraviesa la ciudad. Este cuerpo de agua provee distintos servicios ecosistémicos y se ve afectado por la actividad propia de la urbanización y del entorno productivo rural. No existen investigaciones o planes de monitoreo participativos de la calidad del agua en la zona y son escasos en la región (Mosqueira y Lavaggi, 2020). Se espera aportar a la discusión sobre la importancia de diseñar políticas públicas que favorezcan la participación de actores locales en la definición y abordaje de problemáticas ambientales.

El artículo se desarrolla en tres etapas. En la primera se presenta un marco teórico que da cuenta, por un lado, de las políticas universitarias que se impulsan desde la Universidad de la República (UdelaR, Uruguay) para implementar diferentes estrategias de desarrollo humano y territorial en la región. También se plantean algunos aspectos relevantes a la hora de centrar la atención en los problemas ambientales y la importancia del involucramiento de distintos actores sociales para su comprensión y abordaje. En segundo lugar, se presenta una descripción general de las actividades realizadas y sus principales resultados. Entre estas actividades se destaca un taller multiactoral llevado a cabo en la ciudad de Tranqueras cuyo principal objetivo fue tener un primer acercamiento a las problemáticas ambientales vinculadas a la calidad del agua en esta zona. Además, se pretendió poner en diálogo conocimientos o percepciones locales sobre las posibles causas que afectan los cuerpos de agua. La instancia permitió identificar diferentes elementos de interés y definir de forma colectiva dónde y cómo serían colectadas las muestras de agua. Se realizó una primera salida a campo que permitió a los participantes reconocer equipos de monitoreo in situ y los puntos definidos para las colectas. Por último, se comparten las principales reflexiones que surgen de la experiencia con la intención de aportar al diseño de políticas públicas vinculadas a la gestión participativa del agua, a las estrategias de educación ambiental y al desarrollo local.

\section{Aportes al desarrollo local desde la UdelaR}

Desde el año 2007, la UdelaR viene impulsando diversas iniciativas a partir de un proceso, denominado Segunda Reforma, el cual propone transformaciones importantes en la institución. Entre las propuestas, y para los fines del presente trabajo, cabe resaltar tres políticas universitarias que posibilitaron este proyecto. Por un lado, aquellos esfuerzos orientados al fortalecimiento de la UdelaR en el interior del país. Entre estas estrategias de transformación sobresale la creación de Centros Universitarios Regionales ${ }^{1}$ concebidos como estructuras 
académicas, con una impronta interdisciplinaria, capaces de dinamizar la actividad social, cultural, económica y productiva de diferentes regiones del país (Rectorado, 2010a). En los centros se crearon Polos de Desarrollo Universitario cuyo objetivo fue impulsar la radicación de grupos docentes de alta dedicación y de alto nivel académico para contribuir en el desarrollo de las funciones universitarias en diferentes regiones del interior del país, atendiendo a su vez necesidades locales. ${ }^{2}$ Una segunda política de relevancia fue la creación de un servicio, el Espacio Interdisciplinario, ${ }^{3}$ que tiene como cometido principal la promoción de encuentros entre diferentes disciplinas para el abordaje de problemas que requieren soluciones que van más allá del ámbito disciplinar (Rectorado, 2010b) y que financió parte de este proyecto. En tercer lugar, se puede considerar a la extensión universitaria, la cual desde la concepción de la extensión crítica y reivindicando los ideales latinoamericanos de universidad, tomó un nuevo impulso dirigiendo esfuerzos para la creación y la consolidación de Unidades de Extensión en todos los servicios y regiones del país (Rectorado, 2010c; Red de Extensión, 2019).

Se promovió una serie de políticas con el objetivo de revitalizar y renovar la enseñanza superior considerando a la extensión, en diálogo con la investigación, como componente fundamental de la enseñanza. Estas políticas se orientaron hacia el desarrollo humano sustentable e impulsaron la integración de diferentes saberes, el abordaje de problemas complejos así como la búsqueda colectiva de soluciones a problemas locales y situados (Arocena, Bortagaray y Sutz, 2008). De esta manera, se desplegaron diferentes estrategias para que la UdelaR trabaje anclada en territorios y a partir de nuevos procesos que vinculen la formación universitaria a la resolución de problemas y cooperación entre diversos actores.

Asimismo, se fomentaron prácticas integrales, entendidas como aquellas experiencias que permiten la articulación e integración de funciones universitarias y potencian el trabajo colectivo desde una perspectiva inter o transdisciplinaria. Estas iniciativas promueven la participación de estudiantes en el abordaje de problemas sociales y la generación de encuentros y diálogos bidireccionales entre diversos actores sociales para el abordaje de una temática concreta, un territorio o un problema en particular (Arocena et al., 2011). Desde el modelo de extensión crítica, se le imprimen ciertas características éticas, políticas, pedagógicas, metodológicas y epistemológicas, con el principal objetivo de contribuir a la resolución de problemas de interés social. Este modelo retoma elementos de la pedagogía de la educación popular freiriana y de la investigación participativa falsbordiana, dos grandes referentes del pensamiento latinoamericano (Tommasino y Cano, 2016). Por ello, se puede señalar que los procesos de extensión tienen

"el potencial de redimensionar los procesos de investigación y enseñanza, vinculando directamente a la universidad con problemas sociales reales y definidos de forma colectiva con la comunidad, aportando así a una mejora en la calidad de vida de la población y a los procesos de coproducción de conocimiento". (Lázaro, et al., 2021, p. 191)

2) Más información en https://www.cci.edu.uy/polos-de-desarrollo-universitario\#: :text=El\%20programa\%20Polos \%20de\%20Desarrollo,la\%20Universidad\%20de\%20la\%20Rep\%C3\%BAblica\%20(

3) El presente artículo surge de las reflexiones sobre una experiencia realizada en el marco del desarrollo de las líneas de investigación y extensión del Laboratorio de Química Biológica Ambiental del CENUR Noreste y, particularmente, en el contexto del proyecto "Cuerpos de agua de la ciudad de Tranqueras: aportes para el abordaje participativo de una problemática socioambiental" financiado por el Espacio Interdisciplinario (https://www.ei.udelar.edu.uy/) en el programa de apoyo a investigaciones interdisciplinarias para estudiantes de grado. Las autoras del presente trabajo agradecen al Espacio Interdisciplinario por su apoyo y financiación, y a Iriana Villagrán por sus aportes al proceso. 
Bajo esta concepción la educación no es vista como una acción de transferencia del saber, sino que es diálogo y comunicación a partir de un encuentro de sujetos interlocutores que buscan la significación de los significados (Freire, 1974). En este sentido, no alcanza con llevar un conocimiento técnico o cultural a una comunidad, sino que implica el diálogo y la integración de distintos saberes tanto como la incorporación de conocimiento y la experiencia de la comunidad local. Así, la perspectiva de la extensión crítica refiere a procesos de intercambio de saberes, de enseñanza-aprendizaje bidireccionales y de generación colectiva de conocimiento. Estos procesos, entre otras posibilidades, contribuyen a la resolución de problemas ambientales, caracterizados por su complejidad, multidimensionalidad y multiescalaridad (González et al., 2016).

\section{Ambiente y problemas complejos}

El concepto de "ambiente", además de tener diversos significados a través del tiempo, involucra distintas dimensiones y problemáticas que se entrelazan con el espacio geográfico $^{4}$ y el territorio, ${ }^{5}$ en tanto se pone el foco en la relación sociedad-naturaleza (Arzeno, 2018). A partir de los años 90 , dada la emergencia entre la problemática ambiental, la economía global y la mundialización de la cultura, se comenzó a repensar la relación entre sociedad, naturaleza y territorio. Los análisis ambientales comenzaron a integrar otros factores, aceptándose progresivamente que los temas ambientales son una cuestión de poder y conducen a desigualdades y diferencias entre grupos y clases sociales. Mientras que unos pocos acceden a los bienes naturales para aprovechar su riqueza, los más desfavorecidos deben soportar en sus entornos las consecuencias de la degradación ambiental (Gazzano y Achkar, 2013).

Gazzano y Achkar (2013) definen el ambiente como un "sistema ambiental" integrado por una red interactiva de estructuras emergentes que funcionan como una totalidad organizada. Este sistema es definido por las interrelaciones entre procesos físicos, químicos, biológicos, sociales, culturales, productivos, tecnológicos, económicos y políticos, a distintas escalas territoriales y temporales. La complejidad de los sistemas ambientales está dada por la heterogeneidad, interdefinibilidad y mutua dependencia de las funciones que cumplen sus elementos. Este marco de análisis caracteriza a los problemas ambientales como el resultado de la falta de comprensión de la naturaleza compleja del ambiente y por una forma particular de apropiarse del mundo. Desde este punto de vista, las causas y consecuencias son múltiples e inciertas y las soluciones no tendrán efectos inmediatos. Para comprender las complejas interacciones entre los elementos del ambiente en un tiempo y

4) Entendido como aquello que es socialmente producido por las acciones sociales, las relaciones y las prácticas, a la vez que forma parte de ellas produciéndolas. Cuando se habla del espacio geográfico se deben considerar tres dimensiones a la vez: el espacio de la imaginación y de lo simbólico; el espacio concebido, conceptualizado y construido discursivamente por profesionales; y el espacio percibido física y empíricamente, resultado de la actividad humana. A partir de ello, se pueden identificar otros elementos que interactúan con el espacio geográfico, tales como territorio, lugar, paisaje, región y ambiente. Cada uno de estos conceptos se centra en diferentes aspectos o problemáticas (Arzeno, 2018).

5) Una forma de entender los territorios es a partir de relaciones de poder que lo definen, delimitan y cualifican (Arzeno, 2018). Lo que se percibe y analiza en un territorio es construido y reelaborado en forma permanente, convirtiéndose en una categoría densa que contiene a la historia que lo contiene (Gazzano y Achkar, 2013). 
espacio dados, se requiere de estrategias y metodologías que permitan la integración de diversas visiones y formas de conocimiento (Gazzano y Achkar, 2013).

Para profundizar algunas cuestiones relevantes del presente trabajo se hace imprescindible señalar los principales aspectos y desafíos que caracterizan a las problemáticas ambientales contemporáneas. Desde mediados del siglo XX, el uso, apropiación, transformación y consumo de los bienes naturales se aceleró a un ritmo y a una escala abrumadores, sin precedentes. Los problemas ambientales emergen de la relación sociedad-naturaleza problematizada por una sociedad en contextos históricos y geográficos precisos. Por tal motivo, se tornan "tema de preocupación y debate en diversos ámbitos: entre los grupos afectados por sus consecuencias negativas, entre investigadores de diferentes disciplinas, en los medios de comunicación, en los gobiernos y el ámbito político" (Merlinsky et al., 2018, p. 16).

A partir del trabajo de Merlinsky et al. (2018) se entiende la necesidad de que la sociedad pueda cuestionar ciertas situaciones particulares como fuentes de daño o peligro (construcción social del riesgo). Para ello se precisa la elaboración o apropiación de conocimientos, el debate de valores y prioridades sociales, de la forma de distribución de los beneficios y de los costos de las actividades que generan los impactos. Algunos grupos cuentan con los conocimientos e información necesarios para comprender la situación o problemática, mientras que otros requieren desarrollar procesos que permitan la construcción del problema. En estos casos, cobran relevancia las argumentaciones elaboradas por las poblaciones afectadas en asociación con otros actores sociales (incluidos grupos del ámbito científico-técnico) y el reconocimiento de las desigualdades de poder, en el sentido de acceso al conocimiento y la posibilidad de participación en la toma de decisiones.

Existen diversas estrategias que hacen factible la generación colectiva de conocimiento a partir del abordaje integral de problemas concretos, como los ambientales. La investigación participativa es una de las posibilidades dentro de la amplia gama de estrategias participativas. En esta estrategia, la cual se presenta en la siguiente sección, resulta muy valiosa para para el análisis, comprensión y acción frente a problemas ambientales (Herrera y López, 2014; Trimble, Iribarne y Lázaro, 2014). La investigación y la extensión se integran en la praxis brindando un enfoque integral a la resolución de problemas complejos.

\section{Monitoreos participativos como una forma de investigaciones transdisciplinarias}

Para intentar comprender esa totalidad compleja de los sistemas ambientales desde ciertos enfoques y marcos epistemológicos se proponen nuevas hipótesis y esquemas cognitivos que promueven articulaciones organizativas o estructurales entre disciplinas separadas que traspasan las fronteras disciplinares (Morin, 2010). Esta forma de reconocer la dinámica y complejidad del ambiente implica moverse hacia marcos epistemológicos y estilos de actividad científica diferentes del tradicional (Funtowicz y Ravetz, 2000). Algunos autores plantean la importancia de avanzar desde marcos reduccionistas hacia otros que atiendan la complejidad de los fenómenos. Señalan que el conocimiento científico es necesario para mejorar los esfuerzos del abordaje de problemáticas ambientales, sin embargo, la fragmentación en su generación hace que algunas ciencias tengan dificultades para integrar sus conocimientos. Uno de los problemas radica en que las ciencias ecológicas y sociales se han desarrollado de manera independiente y no se combinan fácilmente (Ostrom, 2009; Morin 2010). La com- 
plejidad de ciertos problemas requiere de la interacción entre saberes, enfoques y grupos de actores en variadas escalas espaciales y temporales. Para ello, es necesario el desarrollo de enfoques que promuevan el diálogo, la reflexión y la participación de distintos colectivos a lo largo del tiempo (Lázaro et al., 2021).

Se plantea entonces la idea de que muchos de los problemas que enfrentan las sociedades contemporáneas, entre ellos los ambientales, deben ser abordados de manera transdisciplinaria. Esta puede entenderse como la formulación de problemas y de propuestas para comprenderlos y resolverlos mediante la interacción de profesionales de diferentes disciplinas con agentes no académicos que pueden aportar otros tipos de conocimientos relevantes. Esta interacción de los actores puede llevar a la conformación de redes sociales de innovación cuya potencialidad es la generación de conocimiento y la transformación de la realidad (Olivé, 2011; Lang, et al., 2012; Olivé, Villamar y Anyul, 2018). En la investigación transdisciplinaria se integran metodologías, herramientas, disciplinas y saberes no académicos, y se incorporan puntos de vista, cosmovisiones, valores y tipos de conocimiento, lo cual aumenta la posibilidad de innovar y de diseñar estrategias. En este marco, las acciones se orientan hacia la búsqueda de soluciones integrales, el aumento de la participación de los implicados, la definición conjunta de los problemas a abordar, así como el diálogo de diferentes saberes. Además del nuevo conocimiento colectivo generado, existen resultados del proceso que son intangibles como el aprendizaje social o el fortalecimiento de capacidades para la toma de decisiones. Estos aspectos son relevantes dado que pueden mejorar las condiciones sociales para la aplicación de los resultados (Gibbons, et al., 1994; Lang et al., 2012; O'brien, Marzano, \& White, 2013; Trimble et al., 2014; Lázaro et al., 2021). Por su parte, la participación exige el desarrollo de mecanismos debidamente establecidos en los cuales se puedan integrar los miembros de la comunidad, los tomadores de decisiones y otros actores no científicos en el proceso de la investigación (Olivé, 2004; 2011).

Una de las formas en que se desarrollan las investigaciones transdisciplinarias son las investigaciones participativas, las cuales son un conjunto de enfoques o estrategias que aparecen en la literatura con distintas denominaciones. Entre las denominaciones más conocidas se encuentran la Investigación Participativa, Investigación-Acción, InvestigaciónAcción Participativa (IAP), Investigación de Base Comunitaria, Investigación Transdisciplinaria, Investigación Colaborativa e Investigación Militante (Brzeski, Graham, y Newkirk, 2001; Balcazar 2003; Sirvent, 2008; O'brien, Marzano, y White, 2013; Herrera y López, 2014; Trimble et al., 2014). Se han desarrollado a través de los últimos 80 años y presentan marcado legado latinoamericano desde mediados del siglo XX, cuando el colombiano Orlando Fals Borda propuso la IAP como estrategia de transformación social. Convergen en su propuesta el pensamiento de grandes intelectuales latinoamericanos de la época, como Paulo Freire, Fernando Cardoso, Enzo Faletto, Celso Furtado, Camilo Torres y Pablo González Casanova, entre otros (Fals Borda \& Rodrígues Brandao, 1991; Devés, 2003; Herrera y López, 2014). Si bien se reconocen raíces históricas y políticas diferentes, actualmente comparten ciertas características metodológicas y epistemológicas. Estas estrategias de investigación han sido utilizadas en distintas áreas tanto a nivel político, social y sanitario, como en la gestión comunitaria de recursos naturales, la producción rural, la pesca artesanal, entre otras (Laurell, 1984; Fals Borda \& Rodrígues Brandao, 1991; Brzeski et al., 2001; Tomasina \& Stolovas, 2009; Trimble et al., 2014; Bouzas, Díaz e Iribarne, 2020; Mosqueira y Lavaggi, 2020). 
Asimismo, cabe señalar que en Latinoamérica la IAP ha tenido un fuerte vínculo con la educación para adultos y popular. Esta estrategia, desde un punto de vista pedagógico, habilita a trabajar a partir de problemas cotidianos, analizando críticamente la realidad, permitiendo generar ciclos de descubrimiento y comprensión de diversas dimensiones de los problemas. La acción puede ser una fuente de nuevos conocimientos, posibles soluciones, nuevas hipótesis, y nuevas preguntas de investigación (Sirvent, 2008).

En el marco de los problemas ambientales, las investigaciones participativas podrían ser estrategias adecuadas para implementar procesos de educación ambiental desde una mirada crítica. Podrían desarrollar acciones para evitar o mitigar daños hacia los ecosistemas más valorados por la población, prever impactos respecto de lo que pudiera efectuarse en ellos e integrar para ello los saberes de las poblaciones locales sobre el estado de situación del territorio. Al abordar un problema de forma integral, articulando la investigación y la extensión, se aporta también al desarrollo de procesos de aprendizaje social, empoderamiento de las comunidades y mejoras en su calidad de vida. En este contexto, es importante destacar que la extensión universitaria presenta gran relevancia y potencialidad, ya que hace factible redimensionar los procesos de coproducción de conocimiento, la integración de conocimientos, y aporta al desarrollo local (Bouzas et al., 2020).

En tanto, cabe considerar que los monitoreos participativos son en esencia investigaciones participativas con ciertas características propias, donde se conjugan estrategias metodológicas así como intereses sociales y políticos. En términos generales, se puede definir al monitoreo participativo como un proceso de coproducción de conocimiento donde se realiza la observación sistemática de parámetros, fenómenos o indicadores que son definidos por un grupo integrado por distintos actores de un territorio, o un grupo transdisciplinario, quienes participan del diseño, planificación e implementación de las acciones durante todo el proceso (Fernandes Couto et al., 2021; Ulloa et al., 2021).

\section{La problemática del agua en Tranqueras}

El departamento de Rivera está ubicado en la región noreste de Uruguay. Limita con los departamentos de Salto, Artigas, Cerro Largo y Tacuarembó, y posee una extensa zona de frontera con Brasil. Ocupa una superficie de $9370 \mathrm{~km}^{2}$ y tiene una población de 103493 habitantes (INE, 2011). En parte del departamento yace el Sistema Acuífero Guaraní (SAG), un reservorio de aguas subterráneas compartido por Uruguay, Paraguay, Brasil y Argentina (Montes, 2007). En el territorio uruguayo ocupa una superficie de $36170 \mathrm{~km}^{2}$ y es el acuifero uruguayo de mayor extensión y potencial productivo. Dentro del departamento de Rivera, las ciudades de Rivera y Tranqueras utilizan al SAG como fuente de agua potable (PNUMA, 2009).

La ciudad de Tranqueras se ubica al noroeste de Rivera y es la segunda ciudad más poblada de este departamento con aproximadamente 7236 habitantes, según el último censo de población realizado (INE, 2011). Además del SAG, la ciudad de Tranqueras es atravesada por el río Tacuarembó, que es el principal cuerpo de agua superficial en la zona. En los últimos años, la ciudad comenzó un plan de saneamiento para las viviendas particulares dado que no contaba en su totalidad con uno adecuado, y en 2017 se aprobó la ordenanza que regula su política de implementación (Decreto Intendencia de Rivera № 1771/017). La disposición final de los residuos domésticos de esta ciudad se hace en un vertedero a cielo abierto situado 
a 200 m del río Tacuarembó. A su vez, existen testimonios de los vecinos que mencionaron haber destinado el pozo de extracción de agua de consumo humano para la realización del pozo negro donde se desagotan las aguas servidas de la vivienda. Ante esta situación de la ciudad, emerge la preocupación sobre la calidad del agua del río Tacuarembó por el posible vertido de aguas cloacales provenientes de hogares sin saneamiento y su percolación al SAG. Hay entonces varios factores que podrían ocasionar un impacto sobre los cuerpos de agua de la zona e implicar un riesgo ambiental y para la salud de los pobladores de Tranqueras (PNUMA, 2009).

Es de destacar que no se conocen antecedentes de investigaciones participativas orientadas a comprender de forma colectiva el estado de la calidad de agua de esta zona. El presente trabajo constituye, en este sentido, un primer esfuerzo por acercarse a los diferentes actores que hacen al territorio y conocer cuáles son aquellos problemas identificados por ellos que podrían estar afectando la calidad de agua.

\section{Metodología}

No existen antecedentes de trabajos con enfoques participativos para abordar problemáticas ambientales vinculadas a la calidad del agua en la ciudad de Tranqueras. Por lo tanto, la premisa fue recoger la preocupación de algunos habitantes de la ciudad con relación a los posibles problemas ambientales de la zona. A su vez, la escasez de información pública de estudios ambientales del lugar promovió la iniciativa de generar un grupo de trabajo conjunto entre la UdelaR y un grupo de ciudadanos residentes en Tranqueras. En este marco, vale destacar que uno de los aspectos más importantes de la descentralización de la UdelaR es el surgimiento del intercambio entre actores universitarios y pobladores de las distintas localidades de la región.

Por lo anterior, y para dar inicio a un proceso participativo, se realizaron entrevistas exploratorias a algunos actores claves de la ciudad. Fueron entrevistados el alcalde, ${ }^{6}$ el director del área ambiental del gobierno departamental, el director de un área protegida de la zona, una trabajadora del área salud de una policlínica local, una administrativa de la junta local, varios docentes de educación media de diferentes disciplinas y varios estudiantes de educación media. El objetivo principal de las entrevistas fue tener un acercamiento a la percepción de la problemática ambiental de Tranqueras desde los distintos actores.

\section{Taller como dispositivo para procesos participativos en temas ambientales}

Una vez realizadas estas entrevistas, se los convocó (a los entrevistados y a otros sugeridos por estos) para implicarse en el proceso del monitoreo participativo de la calidad de agua del río Tacuarembó en el tramo que atraviesa la ciudad. Este proceso tuvo como dispositivo principal la organización y desarrollo de un taller multiactoral, en el cual se destaca la

6) El sistema de gobierno uruguayo está integrado por tres niveles: nivel nacional (primer nivel de gobierno) representado por tres poderes (Ejecutivo, Legislativo y Judicial) y otras personas públicas; el nivel departamental (segundo nivel de gobierno) representado por gobiernos departamentales a través de las intendencias y la Junta Departamental; y el nivel municipal (tercer nivel de gobierno) dedicado a asuntos locales a través de un Concejo Municipal que es presidido por un alcalde. 
participación de estudiantes y docentes de educación media, en su mayoría de la UTU ${ }^{7}$ de Tranqueras. El taller realizado puede considerarse como un espacio de aprendizaje social donde "se trabaja, se crean obras, se comparten conocimientos, se esculpen nuevas formas, y se reparan barcos para emprender nuevos viajes" (Cano, 2012, p. 31). De esta forma, y siguiendo los principios que sustentan al taller como metodología de trabajo colectivo en torno a un problema en común, se planteó la utilización de determinadas técnicas en tres etapas diferentes (Tabla 1): 1) presentación del proyecto y pautas de trabajo; 2) intercambio de saberes en tres subgrupos a partir de consignas; 3) mapeo colectivo de los lugares para la colecta de agua a muestrear.

Tabla 1. Estructura del taller diseñado en tres etapas y con una facilitadora del diálogo

\begin{tabular}{|c|c|c|}
\hline Etapa & Actividades & Objetivos \\
\hline 1. Apertura & $\begin{array}{l}\text { - presentación de los participantes, } \\
\text { - presentación de pautas de diálogo y rol del facilitador, } \\
\text { - presentación del proyecto, } \\
\text { - intercambio con participantes. }\end{array}$ & $\begin{array}{l}\text { En esta etapa se espera un primer acercamiento entre los } \\
\text { participantes y el equipo de trabajo. La presentación de las } \\
\text { reglas de diálogo permite dar marco a la forma esperada del } \\
\text { intercambio. }\end{array}$ \\
\hline $\begin{array}{l}\text { 2. Intercambio } \\
\text { y discusión }\end{array}$ & $\begin{array}{l}\text { - trabajo en sugbrupos (3) con diferentes consignas, } \\
\text { - presentación de trabajos en plenaria, } \\
\text { - discusión de los resultados de cada grupo. }\end{array}$ & $\begin{array}{l}\text { Se espera relevar la información de cada participante. Cada } \\
\text { etapa permite el intercambio, integración y ajuste/corrección } \\
\text { de la información que es plasmada en papelógrafos. }\end{array}$ \\
\hline $\begin{array}{l}\text { 3. Mapeo co- } \\
\text { lectivo y acuer- } \\
\text { dos finales }\end{array}$ & $\begin{array}{l}\text { - presentación de contaminantes de cursos de aguas } \\
\text { y presentación de métodos y técnicas para monitorear, } \\
\text { - fijar puntos de monitoreo. Cada grupo selecciona } \\
\text { sitios en el mapa de posibles nuestros y justifica. }\end{array}$ & $\begin{array}{l}\text { En esta etapa se espera un acercamiento a técnicas para } \\
\text { evaluar la calidad de agua así como acuerdos para definir } \\
\text { colectivamente los sitios del muestreo. }\end{array}$ \\
\hline
\end{tabular}

Para finalizar, se realizó una ronda de evaluación individual de la actividad. Las herramientas participativas trabajan a partir de la experiencia de las personas que participan y están basadas principalmente en el diálogo y en técnicas visuales. De este modo, todos los participantes son considerados como fuente de información y agentes para la toma de decisiones, para analizar los problemas y para contribuir a las soluciones (Geilfus, 2002).

\section{Resultados del proceso}

A continuación se presentarán los resultados a partir del análisis del taller y de las actividades posteriores al mismo.

\section{Análisis del taller y rol del facilitador del diálogo}

Dos elementos definen metodológicamente este taller: la estructura del dispositivo y la facilitación del diálogo. El taller, concebido como un "dispositivo" en el sentido propuesto por Cano (2012) plantea un trabajo en y con grupos para lograr un efecto donde "cobrarán importancia las relaciones entre lo verbal y lo no verbal, lo dicho y lo no dicho, los discur-

7) Sigla con la cual es conocida la institución a cargo de la educación técnica y tecnológica de nivel medio y terciario no universitario así como de la formación profesional (básica y superior) en todo el territorio de la República Oriental del Uruguay. Más información: https://www.utu.edu.uy/utu/institucional/presentacion 
sos y las prácticas" (Cano, 2012, p. 36). Fue planificado para generar "las condiciones de posibilidad, que provoca o pone en visibilidad y eventualmente en enunciabilidad latencias grupales, institucionales o comunitarias" (Fernández, 2007 en Cano 2012, p. 36).

Si bien la convocatoria para esta actividad fue amplia e incluyó a autoridades locales (por ejemplo, el alcalde) fue el sector educativo el que se vio mayormente representado. Varios docentes de educación media (bachillerato y UTU), en consulta previa con el equipo organizador, optaron por participar acompañados por algunos de sus estudiantes. Así, participaron de la actividad profesoras y profesores de enseñanza media de diferentes disciplinas, estudiantes de nivel preuniversitario, una integrante de la ONG local vinculada a temáticas ambientales y el director de un área protegida cercana. De esta forma se logró la conformación de un grupo de referencia integrado por residentes de la zona (diferentes edades, géneros, ocupaciones) comprometidos e interesados en la temática.

Como se mencionó, el taller fue organizado en tres etapas, según se observa en la Tabla 1. Siguiendo a Cano (2012), se podría decir que contribuyó como dispositivo de trabajo grupal. Fue planificado en un tiempo limitado y se realizó con determinados objetivos particulares. Así fue posible desarrollar un proceso pedagógico sustentado en la integración teórico-práctica, donde el protagonismo de los participantes, el diálogo de saberes y la producción colectiva de aprendizajes, intervinieron en las personas participantes y en la situación de partida.

Las etapas del taller fueron pensadas para contribuir a la comprensión del problema y a las técnicas de laboratorio que serían utilizadas para evaluar la calidad del agua. La primera etapa, a cargo del equipo organizador, considerado un necesario punto de partida, fue para la socialización de conocimientos provenientes de la academia. La segunda etapa se organizó como un espacio de intercambio y discusión de diferentes problemáticas. Para ello se conformaron tres subgrupos de trabajo que reflexionaron e intercambiaron sus conocimientos a través de preguntas que fueron propuestas a cada subgrupo por el equipo organizador. Las preguntas de las consignas fueron pensadas con la finalidad de promover un diálogo crítico sobre la temática por parte de los participantes. En la Tabla 2 se observan estas preguntas junto con los principales resultados.

Tabla 2. Preguntas realizadas durante el trabajo en subgrupos y principales resultados

\begin{tabular}{|c|c|}
\hline Consigna & Principales resultados \\
\hline $\begin{array}{l}\text { ¿Qué posibles fuentes contaminantes, } \\
\text { antropogénicas o naturales, identifi- } \\
\text { camos sobre los cursos de agua de } \\
\text { Tranqueras (medio rural y urbano)? }\end{array}$ & $\begin{array}{l}\text { - Basurero municipal: está próximo al río; faltan lugares donde depositar los residuos sin conta- } \\
\text { minar el agua. } \\
\text { - Producción de madera: contaminación natural (materia orgánica) y antropogénica (procesos } \\
\text { productivos). } \\
\text { - Problemas sanitarios: falta de saneamiento en los centros urbanos. } \\
\text { - Uso de agroquímicos: producción de sandía y forestal. }\end{array}$ \\
\hline $\begin{array}{l}\text { ¿Qué influencia podría tener la conta- } \\
\text { minación de los cursos de agua super- } \\
\text { ficiales de Tranqueras, sobre la reserva } \\
\text { subterránea del Acuífero Guaraní? }\end{array}$ & $\begin{array}{l}\text { El origen de la contaminación podría ser la producción agrícola, el basurero, los pozos negros } \\
\text { y las aguas servidas. El problema es multifactorial, depende de situaciones geomorfológicas y } \\
\text { el tipo de contaminante. Concluyen que la contaminación puede tener impactos negativos en } \\
\text { el acuífero. }\end{array}$ \\
\hline $\begin{array}{l}\text { ¿Cómo se mide en Tranqueras el posi- } \\
\text { ble impacto ambiental (análisis, moni- } \\
\text { toreo, etc.)? }\end{array}$ & $\begin{array}{l}\text { Señalan que la OSE y la Intendencia Departamental realizan estudios, pero desconocen en qué } \\
\text { consisten. Identifican un lugar donde se realizan baños recreativos y pesca artesanal en el que } \\
\text { no se hacen estudios (“La Olla”). }\end{array}$ \\
\hline
\end{tabular}

Fuente: elaboración propia, 2021 
La tercera y última etapa implicó la socialización de los intercambios de los grupos así como el diseño colectivo del primer muestreo. Se identificaron diferentes puntos sobre el río Tacuarembó, aguas arriba, previo a su paso por la ciudad de Tranqueras (control), asociados a actividades actuales y pasadas y aguas abajo de la ciudad. En esta etapa la deliberación fue la clave, entendida esta como un intercambio de razones o argumentaciones orientadas al entendimiento común. Inicialmente fueron propuestos ocho puntos para los muestreos. Durante el intercambio se propició la reflexión y la argumentación acerca de por qué algunos puntos no sería viable o necesario muestrear. Este tipo de espacios de deliberación espera obtener como resultado un consenso argumentado que permita un movimiento desde posturas personales a posturas colectivas (Lázaro et al., 2018; García, 2020). De esta forma, después de discutir cada sitio, de explicar por qué se había definido tal o cual punto, las posibilidades reales de colectar agua, así como los argumentos sobre por qué debía muestrearse, se definieron de manera colectiva seis sitios de colecta: antes de ingresar a la ciudad como sitio control, transcurriendo en la ciudad para conocer si existen impactos urbanos vinculados a ciertos usos, y luego de ella para conocer si existen cambios sustantivos globales por aportes urbanos (Figura 1). Para definir el sitio control se analizó entre los diferentes participantes cuál era el lugar que ofrecía información sobre el estado de la calidad del agua previo al pasaje del río por la ciudad de Tranqueras. Al considerar un estado previo al transcurso del río por la ciudad, se pueden comparar variaciones entre los parámetros de calidad de agua entre distintos sitios de muestreo y considerar posibles afectaciones vinculadas a las actividades de la zona. En el caso de encontrar resultados alterados, se podría inferir que el aporte de sustancias proveniente del centro urbano genera impactos negativos en la calidad del agua.

Figura 1. Puntos del muestreo del río Tacuarembó

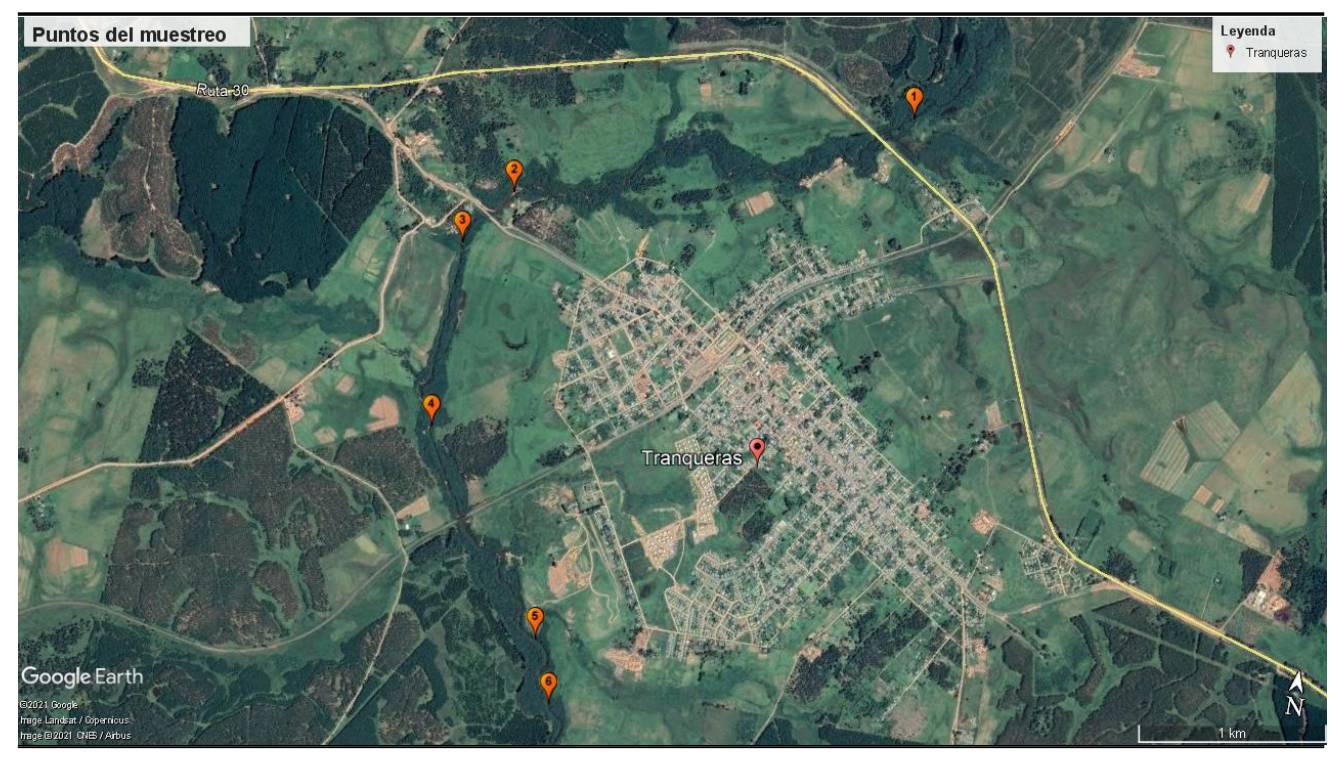

Nota: En el mapa se señalan los seis puntos del muestreo, los cuales fueron definidos de forma colectiva en el taller:

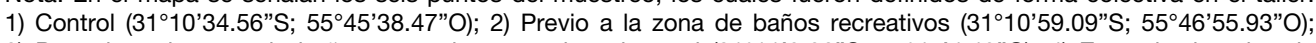

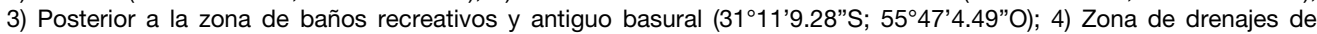
aserraderos (31 $11^{\prime} 41.64^{\prime \prime}$; $\left.55^{\circ} 47^{\prime} 4.25^{\prime \prime}\right)$ ); 5) Zona de la "trinchera sanitaria" donde se encuentra el sitio de disposición

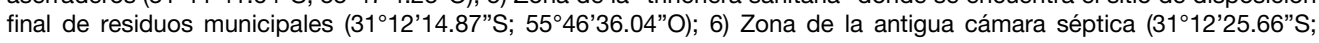
$55^{\circ} 46$ '31.07”O). 
Al finalizar el taller se hizo una evaluación individual de la actividad. Allí cada participante expresó cómo le había resultado el espacio de encuentro. En general, todos acordaron en que fue una instancia positiva e interesante. A partir de la percepción de los participantes se destacó la posibilidad de involucrarse docentes con alumnos y trabajar juntos. También se destacó la oportunidad de desarrollar este tipo de instancias porque se descubren aspectos locales a la vez que se puede reflexionar sobre impactos ambientales en la zona y cómo contribuir como ciudadanos. Este tipo de evaluación por parte de los participantes reafirma la idea de que los procesos de extensión pueden aportar al desarrollo humano y al aprendizaje colectivo, promoviendo con ello el aprendizaje social.

También se señaló que fue novedoso conocer aspectos concretos sobre qué se evalúa y por qué para conocer la calidad de agua de un río. Se consideró importante la posibilidad de involucrar a la comunidad en procesos que convencionalmente son realizados por técnicos. En este caso, se destacó la oportunidad de trabajar con las herramientas de una de las disciplinas involucradas (química ambiental), como un todo independiente de la profesión. En otras palabras, lo que se puso de relieve, y que es importante en los procesos de trabajo participativo, fue la oportunidad de que cualquier ciudadano pueda comprender y aplicar los procesos que tradicionalmente son utilizados por expertos en diferentes áreas disciplinares. Los participantes comprendieron la importancia de los parámetros físicoquímicos utilizados, cómo interpretar los resultados y la relación con la calidad de agua del río. Este aspecto es de interés en los procesos de ciencia ciudadana ya que, por un lado, son elementos valiosos en la dimensión educativa y en los procesos de educación ambiental y, por otro, aportan a la autonomía y pensamiento crítico de la ciudadanía ambiental (Barcia, 2013). En este sentido, el aporte al proceso educativo de todos los participantes, así como de formación de ciudadanía ambiental, es tan relevante como los resultados de las muestras analizadas.

En la misma línea, se indicó la importancia del involucramiento de los estudiantes en temas relacionados con el abordaje o resolución de problemas ambientales de la ciudad. Asimismo, se valoró la instancia y método de trabajo (taller y facilitador) considerando que puede ser muy interesante aplicarlo para otros temas. $Y$ atento a estos comentarios sobre el desarrollo del taller, y de acuerdo con lo planteado por Merlinsky (2018), se podría señalar que el taller permite el acercamiento a la dimensión social de los problemas ambientales. Esta dimensión suele ser el aspecto menos visible en las investigaciones sobre degradación ambiental porque las disciplinas que se ocupan de estos temas muchas veces priorizan el análisis muy especializado de problemas de calidad de agua, suelos y aire, y evitan los conflictos entre los actores sociales.

El taller contó con una facilitadora del diálogo (integrante del equipo), ya que este tipo de procesos requiere asegurar la participación de todos y promover la comprensión mutua en cuanto a propiciar la apertura a las necesidades y visiones de los demás participantes. La facilitación también permite generar un espacio para la construcción de conocimientos y de responsabilidades compartidas. De esta manera, el rol del facilitador es asegurar que se den las condiciones para el intercambio de visiones de forma respetuosa conforme a la estructura y tiempos planificados (Trimble et al., 2014; Lázaro, Bortagaray y Stuhldreher, 2018; García, 2020). 


\section{Actividades posteriores al taller}

Luego de la determinación de los puntos de muestreo, se acordó con parte de los participantes del taller realizar una toma de muestras de agua y determinación de algunos parámetros físicos y químicos dentro de los habitualmente utilizados para caracterizar la calidad del agua. A tal efecto se efectuó el muestreo de los seis puntos definidos colectivamente sobre el río Tacuarembó, en zonas aguas arriba y abajo del recorrido del río por la ciudad y algunos puntos en la zona cercana al casco urbano. Durante la experiencia, los participantes fueron capacitados para hacer la toma de muestras y mediciones in situ de parámetros como $\mathrm{pH}$, temperatura, conductividad y sólidos disueltos totales (Tabla 3). Estos valores están asociados a la calidad del agua, siendo el pH un parámetro que, de no estar dentro de los rangos esperados, puede afectar el ecosistema del cuerpo de agua. Además, tanto la conductividad como los sólidos disueltos totales son una medida de las sustancias orgánicas e inorgánicas presentes en el agua; sus valores alterados denotan un posible aporte de contaminantes en la zona. Todos los parámetros determinados en este muestreo se encontraron dentro de los rangos aceptables para un cuerpo de agua con las características de uso del río Tacuarembó (Decreto 253/79).

Las muestras colectadas en colaboración de los estudiantes participantes fueron transportadas al Laboratorio de Química Biológica Ambiental del CENUR Noreste para su procesamiento y conservación para posteriores estudios. Esta etapa también fue formativa para los participantes ya que comprendieron qué tipo de planificación y cuidados deben tenerse en cuenta en el proceso de un monitoreo. Se destaca la importancia de cuidar la calidad tanto del proceso de participación como de la colecta de muestras y su posterior análisis.

Tabla 3. Parámetros fisicoquímicos determinados en los seis puntos del muestreo participativo en el río Tacuarembó

\begin{tabular}{|c|c|c|c|c|c|c|}
\hline & \multicolumn{6}{|c|}{ Puntos de muestreo } \\
\hline & 1 & 2 & 3 & 4 & 5 & 6 \\
\hline Temperatura $\left({ }^{\circ} \mathrm{C}\right)$ & 23,1 & 22,9 & 22,7 & 22,8 & 23,5 & 22,5 \\
\hline Sólidos disueltos totales (ppm) & 6,05 & 5,91 & 6,13 & 6,11 & 5,71 & 5,57 \\
\hline Conductividad $(\mu \mathrm{S} / \mathrm{cm})$ & 9,45 & 9,23 & 9,57 & 9,55 & 8,93 & 8,73 \\
\hline
\end{tabular}

Fuente: elaboración propia, 2021

Con posterioridad a la instancia de muestreo y laboratorio, un integrante del grupo de referencia consideró que los resultados del trabajo debían ser compartidos en centros educativos. Por este motivo, y como una forma de aportar elementos para la comprensión ciudadana, se realizó un taller con estudiantes del último año de educación media donde se expuso la experiencia de trabajo con el grupo. Los estudiantes que participaron de esta instancia era la primera vez que tomaban contacto con una experiencia participativa en su localidad, lo cual generó interés por continuar involucrados en el estudio de las problemáticas ambientales de su ciudad. En esa instancia se subrayó la importancia de la participación ciudadana en los estudios ambientales de la zona para aportar a la formación de la comunidad en estos temas y fomentar su interés. Este aspecto es relevante como una oportunidad 
de generación de un espacio de enseñanza-aprendizaje mutuo, una de las características o resultados destacables (y deseados) de los procesos participativos, de los procesos de educación ambiental y, en particular, de los procesos de extensión universitaria.

\section{Reflexiones finales}

A partir de esta experiencia se destaca el efecto que genera en los participantes la visualización conjunta de las posibles causas de problemas ambientales de su localidad. La realización de investigaciones participativas fomenta el intercambio de conocimientos y vivencias entre los miembros de una comunidad sobre temáticas de interés. En este caso, se pueden señalar algunos aprendizajes y también desafíos para futuras acciones.

En primer lugar, nos referimos a los aportes que los procesos participativos pueden brindar al desarrollo local y a la mejora de la calidad de vida de sus pobladores. La región noreste de Uruguay, donde se llevó a cabo esta experiencia, es la zona con los mayores índices de pobreza y menores de desarrollo humano del país. En este sentido, las acciones que puedan ser impulsadas desde la UdelaR, en especial con enfoques integrales, son fundamentales para dinamizar diferentes dimensiones de los territorios y contribuir en su desarrollo. En cuanto a los problemas ambientales, el aporte de los procesos participativos puede contribuir a mejorar el nivel del conocimiento de la problemática local, aumentar la comprensión de sus causas, el interés por minimizar los problemas y empoderar a los ciudadanos frente a la toma de decisiones colectivas.

En segundo lugar, considerando las condiciones de desarrollo humano de la región, se justifica continuar y profundizar el abordaje de los problemas de forma participativa. Esto permitirá la identificación de problemas y formulación de propuestas para comprenderlos y resolverlos mediante la interacción de diversos actores que, aun sin provenir de disciplinas académicas, poseen experiencia y conocimientos importantes con relación al problema. Al respecto, y situándonos en el marco de las comunidades que integran el noreste del país, el hecho de trabajar problemáticas ambientales locales con la población promueve una resignificación en la apropiación de su territorio. Realizar acciones directas sobre problemas ambientales facilita concebir soluciones que generalmente son planteadas como alejadas de alternativas posibles para la comunidad y, a la vez, promueve el aprendizaje social. De esta manera es posible desarrollar estrategias participativas que podrían construirse a partir de una evaluación crítica de la información con el objetivo de aportar a políticas públicas y la gestión/acción de temas concretos. En lo que atañe a políticas públicas en particular, cabe destacar que Uruguay ha impulsado en los últimos años diversas normas jurídicas, mecanismos, planes y programas en los que se incluye la participación ciudadana. Algunos de estos instrumentos están vinculados directamente con la gestión de los recursos hídricos del país (como es el caso del Plan Nacional de Aguas) ${ }^{8}$ y otros se relacionan con la educación ambiental (Plan Nacional de Educación Ambiental). ${ }^{9}$ En este 
contexto, reviste importancia que la participación sea concebida desde enfoques críticos como oportunidades para involucrarse activamente en la planificación y toma de decisiones de diversos asuntos ambientales y no solo como espacios de información y consulta. Crear estos espacios de participación también plantea la necesidad de desarrollar ciertas actitudes y aptitudes sociales, lo cual requiere el fortalecimiento de las capacidades académicas, políticas, comunitarias e institucionales.

En tercer lugar, en cuanto a aspectos metodológicos, cabe destacar la relevancia de la facilitación del diálogo entre los actores con sus distintas visiones, intereses y saberes, tanto en el taller como a través de las instancias que se sucedieron. Este caso en particular tuvo la participación de personas de varias generaciones y con diferentes relaciones de poder entre ellas (por ejemplo, la relación docente-estudiante de educación media). Sin embargo, se observó que el vínculo que se dio entre ellos no fue jerárquico sino colaborativo. Con referencia a las debilidades o desafíos de este tipo de procesos, tienen que ver con aspectos económicos y temporales. Estas estrategias participativas requieren más tiempo que las estrategias de investigación convencional ya que el proceso de vinculación e integración entre diferentes actores es clave. Es necesario implementar más mecanismos de tipo taller para estrechar vínculos, fortalecer el entendimiento mutuo y generar confianza entre las personas que participan. En este sentido, contar con mayor tiempo implica que se cuente con más recursos económicos y humanos.

El aspecto que puede debilitar este tipo de procesos, y que se observó en la presente experiencia, es la forma en que se define la problemática a abordar. En este caso, el problema a investigar fue propuesto por el equipo universitario y no en conjunto con la comunidad, lo cual podría haber afectado la participación o el interés de algunos actores. Por lo tanto, el gran desafío para fortalecer el vínculo universidad-sociedad depende en gran medida de las estrategias utilizadas para la intervención y el tiempo que se sostenga el equipo en el territorio trabajando junto con la comunidad, promoviendo espacios de construcción y reflexión colectiva. Todo ello depende de aspectos económicos, como mencionamos, pues definen el tiempo y calidad del proceso que se pueda llevar a cabo. Pero también depende en gran medida de aspectos metodológicos. Es decir, aquel conjunto de técnicas colaborativas que se introducen para desarrollar el proceso así como la definición del problema de investigación.

Por último, uno de los desafíos tal vez más relevantes en el marco de este trabajo está vinculado a la dimensión epistemológica de los problemas ambientales y la forma en que se genera su conocimiento. De acuerdo con el planteo de Eschenhagen (2010), al abordar problemas ambientales generalmente no se explicitan las bases epistemológicas a partir de las cuales se plantean los acercamientos para el análisis y la construcción del conocimiento. Por ello, cuestionar, confrontar y diferenciar las distintas perspectivas con que se pueden explicar y abordar los problemas ambientales se torna un elemento de relevancia. De igual modo, los problemas ambientales han alcanzado un nivel de complejidad tal que precisan un esfuerzo por parte de todos los actores territoriales implicados en ellos, sean estos académicos o no, para comprender sus causas y transformarlas. En este sentido, el modelo de extensión crítica se complementa con el modelo concientizador, pues propone compartir los bienes, incluidos los educativos y culturales, en forma dialógica y liberadora, creando conciencia entre los participantes. En este contexto y siguiendo a Serna (2007, en 
Escobedo y Astudillo, 2016), concientizarse significa:

"un cambio de mentalidad que implica comprender realista y correctamente la ubicación de cada uno en la naturaleza y en la sociedad; la capacidad de analizar críticamente sus causas y consecuencias y establecer comparaciones con otras situaciones y posibilidades; y una acción eficaz y transformadora. Una consecuencia casi inevitable es la participación política y la formación de grupos de interés y presión". (p. 49)

\section{Referencias bibliográficas}

Arbildi, E. (2017). Estudio de posibles riesgos ambientales en cuerpos de agua del Departamento de Rivera. Tesina de Graduación de Licenciatura en Bioquímica. Facultad de Ciencias. UdelaR.

Arocena, R.; Bortagaray, I. y Sutz, J. (2008). Reforma Universitaria y desarrollo. Tradinco.

Arocena, R.; Tommasino, H.; Rodríguez, N.; Sutz, J.; Álvarez Pedrosian, E. y Romano, A. (2011). Integralidad: tensiones y perspectivas. CSEAM.

Arzeno, M. (2018). Extensión en el territorio y territorio en la extensión. Aportes a la discusión desde el campo de la Geografía. +E Revista de Extensión Universitaria, 8(8), 3-11.

Balcazar, F. (2003). Investigación acción participativa (IAP): aspectos conceptuales y dificultades de implementación. Fundamentos en humanidades, IV(I/II-7/8), 59-77. Universidad Nacional de San Luis.

Barcia, L. (2013). Ciudadanía ambiental: ¿Desafío, herramienta o compromiso ético para la Educación Ambiental? Quehacer Educativo, 50-56.

Bouzas, A.; Díaz, I. e Iribarne, P. (2020). Mapeo de servicios ecosistémicos para la planificación del territorio: una experiencia con abordaje participativo. Tekoporá. Revista Latinoamericana de Humanidades Ambientales y Estudios Territoriales, 2(1), 33-57.

Brzeski, V.; Graham, J. y Newkirk, G. (2001). Participatory Research and CBCRM: In context. Coastal Resources Research Network (CoRR). Dalhousie University.

Cano, A. (2012). La metodología taller en los procesos de educación popular. ReLMeCS, 2,(2), $22-52$.

Cornwall, A. \& Jewkes, R. (1995). What is participatory research? Social Science and Medicine 41(12): 1667-1676.

Decreto Intendencia de Rivera N 1771/017 (2017). Ordenanza Plan de Desarrollo y Ordenamiento Territorial de Tranqueras. Informe Ambiental Estratégico.

Decreto Nacional N²53/79 (1979). Normativa para prevenir la contaminación ambiental, a través del control de las aguas en Uruguay. https://www.impo.com.uy/bases/decretos/253-1979

Devés, E. (2003). El pensamiento latinoamericano en el siglo XX. Tomo II. Desde la CEPAL al neoliberalismo (1950-1990). Centro de investigaciones Diego Barros Arana. 2da. ed. Biblos.

Eschenhagen, M. L. (2010). Los límites de la retórica verde o ¿Por qué después de más de 30 años de esfuerzos no se observan mejoras ambientales sustanciales? Gestión y Ambiente, 13(1), 111-118.

Escobedo, M. y Astudillo, G. (2016). Aportes para la discusión sobre la extensión universitaria. Masquedós, 1(1), 47-59.

Fals Borda, O. y Rodrígues Brandao, C. (1991). Investigación Participativa. Instituto del hombre. Ediciones de la Banda Oriental.

Fernandes Couto, J.; Terezinha Costa, A.; Lima Pereira1, A.; Carolina de Souza Lima, A.; Simões Maciel, A.; Carvalho Lins, F.; Lourenço dos Reis, P. y Lúcia de Miranda, V. Guarda (2021). Avaliação e monitoramento participativo na gestão da qualidade da água. Alemur, 6(1), 1-11.

Freire, P. (1974). La educación como práctica de la libertad. Siglo XXI Editores. 
Funtowicz, S. y Ravetz, J. (2000). La ciencia posnormal: Ciencia con la gente. Icaria.

García, P. (2020). Análisis Causal Estratificado Participativo: una metodología para abordar problemáticas ambientales. Tesina de Grado, Ciencias Biológicas. Facultad de Ciencias. UdelaR.

Gazzano, I. y Achkar, M. (2013). La necesidad de redefinir ambiente en el debate científico actual. Gestión y Ambiente, 16(3), 7-15.

Geilfus, F. (2002). 80 herramientas para el desarrollo participativo: diagnóstico, planificación, monitoreo, evaluación. Instituto Interamericano de Cooperación para la Agricultura (IICA).

Gibbons, M.; Limoges, C.; Nowotny, H.; Schwartzman, S.; Scott, P. y Trow, M. (1994). La nueva producción de conocimiento. La dinámica de la ciencia y la investigación en las sociedades contemporáneas. Ediciones Pomares-Corredor.

González, J.; Miguel, M.; Rosso, I.; Toledo López, A. y Toledo López, V. (2016). Mapeando el barrio construimos territorio. Experiencia de cartografía social en Villa Aguirre, Tandil. Masquedós, 1(1), 61-71.

Herrera, N. y López, L. (Comps.) (2014). Ciencia, compromiso y cambio social. Textos de Orlando Fals Borda. 2da. ed. El Colectivo-Lanzas y Letras, Extensión Libros.

INE (Instituto Nacional de Estadística) (2011). Resultados Censo 2011. Departamento de Rivera. Uruguay.

Lang, D.; Wiek, A.; Bergman, M.; Stauffacher, M.; Martens, P.; Moll, P.; Swilling, M. \& Thomas, C. (2012). Transdisciplinary research in sustainability science: practice, principles, and challenges. Sustaintaible Science, 7(1), 25-43.

Laurell, A. C. (1984). Ciencia y experiencia obrera: la lucha por la salud en Italia. Cuadernos Políticos, (41). Editorial Era.

Lázaro, M.; Iribarne, P.; Adalyiza, P.; Rumeau, D. y López-Echagüe, C. (2021). Análisis participativo del problema de los residuos en Montevideo: aplicación del Análisis Causal Estratificado. Revista Iberoamericana de Ciencia, Tecnología y Sociedad-CTS, 16(46).

Lázaro, M.; Bortagaray, I. y Stuhldreher, A. (2018). Imaginando el futuro en Tacuarembó. Instituto de Desarrol/o Sostenible, Innovación e Inclusión Social. Psicolibros, Waslala.

Merlinsky, G.; Toledo López, V.; Schmidt, M.; Fernández Bouzo, S.; Tobías, M.; Langbehn, L.; Pereira, P. y Capalbo, T. (2018) Defender lo común: qué podemos aprender de los conflictos ambientales. Instituto de Investigaciones Gino Germani. Universidad de Buenos Aires.

Montes, R. (Ed). (2007). Acuífero Guaraní: Avances en el conocimiento para su gestión sustentable. ALHSUD. Morin, E. (2010). Sobre la interdisciplinariedad (pp. 9-15). Publicaciones Icesi.

Mosqueira, A. y Lavaggi, M. (2020). Diagnóstico participativo de la calidad de agua como herramienta para la gestión ambiental. El caso del municipio de Vichadero, Rivera, Uruguay. Tekoporá. Revista Latinoamericana de Humanidades Ambientales y Estudios Territoriales, 2(1), 33-57.

O'brien, L.; Marzano, M. \& White, R. (2013). "Participatory interdisciplinarity": Towards the integration of disciplinary diversity with stakeholder engagement for new models of knowledge production. Science and Public Policy, 40(1), 51-61.

Olivé, L. (2011). Interdisciplina y transdisciplina desde la filosofía. Ludus Vitalis, 19(35), 251-256.

Olivé, L.; Villamar, A. A. y Anyul, M. P. (2018). Interdisciplina y transdisciplina frente a los conocimientos tradicionales. CTS: Revista iberoamericana de ciencia, tecnología y sociedad, 13(38), 135-153.

Ostrom, E. (2009). A General Framework for Analyzing Sustainability of Social-Ecological Systems. Science, (325), 419-422.

PNUMA (Programa de las Naciones Unidas para el Medio Ambiente). (2009). Geo-localidades urbanas de Rivera. PNUD.

Rectorado (2010a). Hacia la reforma universitaria \#1. La Universidad en el interior. Tradinco. 
(2010b). Hacia la reforma universitaria \#8. Espacio Interdisciplinario en construcción. Tradinco.

(2010c). Hacia la reforma universitaria \#10. La extensión en la renovación de la enseñanza: Espacios de Formación Integral. Tradinco.

Red de Extensión (2019). Tejer la red: experiencias de extensión desde los servicios 2008-2018. Servicio Central de Extensión y Actividades en el Medio, UdelaR. Gráfica Mosca.

Sirvent, M. T. (2008). Educación de adultos: investigación, participación, desafíos y contradicciones. Miño y Dávila Editores.

Tomasina, F. y Stolovas, N. (2009). Salud de los trabajadores. En Condiciones laborales y organización de/ trabajo (pp. 35-58). UdelaR. Red Académica de Trabajo. Comisión sectorial de Educación Permanente.

Tommasino, H. y Cano, A. (2016). Avances y retrocesos de la extensión crítica en la Universidad de la República de Uruguay. Masquedós, 1(1), 9-23.

Trimble, M.; Iribarne, P. y Lázaro, M. (2014). Una investigación participativa en la costa uruguaya: características, desafíos y oportunidades para la enseñanza universitaria. Desenvolvimento e Meio Ambiente, 32, 101-117. Ulloa, A.; Godfrid, J.; Damonte, G.; Quiroga, C. y López, A. (2021). Monitoreos hídricos comunitarios: conocimientos locales como defensa territorial y ambiental en Argentina, Perú y Colombia. Íconos Revista de Ciencias Sociales, (69), 77-97. 